\title{
Investigation of the Temperature Dependence of Free Volume in Polymethylpentene by Positron Annihilation Method
}

\author{
A. Danch And W. Osoba \\ Institute of Physics, Silesian University \\ Uniwersytecka 4, 40-007 Katowice, Poland
}

\begin{abstract}
Positron lifetime measurements in polymethylpentene were performed in a wide temperature range. A conventional fast-slow coincidence lifetime spectrometer with plastic scintillators was used. All the measurements were made in vacuum. Additional measurements (X-ray diffraction, differential scanning calorimetry, and mechanical spectroscopy) were also performed to establish properties of samples. Mean free volume radii in the investigated samples were estimated from the positron lifetimes measurements results. An increase in $I_{3}$ with the elapsed time of measurements was observed.
\end{abstract}

PACS numbers: 71.60.+z, 78.70.Bj

\section{Introduction}

Positron annihilation spectroscopy turned out to be a very useful method for investigation of different aspects of polymer properties [1,2]. Many different external conditions can perturb the fate of the positron in polymer matter and due to this, they might change its lifetime. In this paper we present results of the positron lifetime measurements in polymethylpentene $-\left[-\mathrm{CH}_{2}-\mathrm{CH}-\left(\mathrm{CH}_{2}-\mathrm{CH}-\left(2 \mathrm{CH}_{3}\right)\right)-\right]-$. This polymer is one of the polyolefines. It is a semi-crystalline polymer for which five modifications (designed I, II, III, IV, V) were found. The modification I is usually obtained when the sample is prepared by melt process. The other four modifications (II-V) might be prepared from diluted solutions. It is relatively easy to get the samples of different structures and we are going to study them in future. We presented very rough and preliminary results of our measurements in the same material in [3]. 


\section{Experimental}

A conventional slow-fast coincidence spectrometer with plastic scintillators was used. The time resolution of the spectrometer, approximated by two Gaussian curves, was determined by analysing the positron lifetimes in kapton foils. Typical values of the full widths at half of the maximum $\left(\mathrm{FWHM}_{i}\right)$ were: $\mathrm{FWHM}_{1}=258.4 \mathrm{ps},\left(I_{1}=73.58 \%\right) ; \mathrm{FWHM}_{2}=365.9 \mathrm{ps}$. Positron lifetime spectra were accumulated to approximately $0.26 \times 10^{6}$ counts. A positron source $\left({ }^{22} \mathrm{Na}\right.$, about $0.15 \mathrm{MBq}$ ) was sealed between two kapton foils. The source correction was taken into account during numerical evaluations. All of the measurements were performed in vacuum (about $10^{-8} \div 10^{-9}$ mbar at low temperature, and $10^{-4} \div 10^{-5}$ mbar, at room temperature). Dimensions of the cold head and its high vacuum jacket allowed us to install two scintillation detectors at the distance of about $25 \mathrm{~mm}$. It reduced drastically the count rate. We acquired one spectrum, so as to reach the above-mentioned statistics, about 24 hours. A closed cycle helium refrigerator system was used to keep the sample at low temperatures. A digital, microprocessor controlled measurement and a control unit was used to regulate the temperature of the sample. It allowed one to keep the temperature of the sample constant, within $\pm(25 \div 30) \times 10^{-3} \mathrm{~K}$. The temperature range of the measurements was from $8.3 \mathrm{~K}$ to $299 \mathrm{~K}$. We started our measurements at $299 \mathrm{~K}$ and we cooled the sample down to $8.3 \mathrm{~K}$ and then we heated it up to $280 \mathrm{~K}$. The measurements were carried out at $20 \mathrm{~K}$ spaced intervals (in the temperature range from $299 \mathrm{~K}$ to $200 \mathrm{~K}$ and from $120 \mathrm{~K}$ to $8.3 \mathrm{~K}$ ) for the cooling cycle. The same temperature steps were used in the heating cycle (in the temperature range from $30 \mathrm{~K}$ to $110 \mathrm{~K}$ and from $210 \mathrm{~K}$ to $290 \mathrm{~K}$ ). The measurements in the temperature range from $190 \mathrm{~K}$ to $120 \mathrm{~K}$ were performed at $10 \mathrm{~K}$ spaced intervals (during the cooling cycle). The same temperature intervals were used in the heating cycle (in the temperature range from $115 \mathrm{~K}$ to $205 \mathrm{~K}$ ). Our choice of the temperature steps was selected so as to follow some changes in the temperature characteristics. The sample was kept in the newly chosen temperature for 40 to 50 min before we started the new measurement.

The sample was made of $0.5 \mathrm{~mm}$ thick film of polymethylpentene, from Goodfellow. Density of the sample material: $0.835 \mathrm{~g} \mathrm{~cm}^{-3}$, hardness (Rockwell): R85, tensile modulus: $1.5 \mathrm{GPa}$, tensile strength: $25.5 \mathrm{MPa}$. Ten square shaped pieces $\left(17 \times 17 \mathrm{~mm}^{2}\right)$ of the polymethylpentene film were cut down from the polymer sheet and the positron source was sandwiched between them (five squares on each side of the source). The sample did not undergo any additional thermal treatment before the measurement.

Additional measurements were carried out on the sample material: the X-ray scattering diffraction pattern was recorded using the Philips diffractometer, thermal characterization of the samples by differential scanning calorimetry (DSC) was performed using Perkin Elmer DSC7 type and the first heating run (20 deg/min) was recorded (by comparison of the X-ray patterns of the studied 
sample and the ones reported in [4], it was possible to assign the crystalline structure to the I modification), and dynamic mechanical thermal analysis (DMTA) was carried out with the same system as previously $[5,6]$ within a frequency range of $0.01-10 \mathrm{~Hz}$.

\section{Results and discussion}

Positron lifetime measurements were performed on the polymethylpentene sample. The spectra were analysed using the package programs written by Kirkegaard et al. [7]. The total number of counts accumulated in our spectra was relatively small $\left(0.26 \times 10^{6}\right)$ and this was why we decided to analyse them in two-step process. As the first step of approximation two exponential components were fitted to the measured spectra with no constraints on lifetimes and intensities during numerical analysis. This procedure resulted in reasonable $\tau_{2}$ and $I_{2}$ values. Nevertheless it is generally accepted to fit three or four components to the measured spectra for polymer samples. As statistics of the obtained spectra was low we decided to fit three components but a lifetime of one of them was fixed. We fixed the lifetime of the second component, $\tau_{2}$. According to our former measurements we chose $380 \mathrm{ps}$ for the fixed $\tau_{2}$ value.

According to a model proposed by Tao [8] and Eldrup et al. [9], the longest lived component of the positron lifetime spectrum may be correlated with the mean radius of the free volume cavity in the polymer matter. They proposed a following equation:

$$
\tau_{3}=0.5[1-R /(R+0.1656)+1 /(2 \pi) \sin (2 \pi R /(R+0.1656))]^{-1},
$$

where $\tau_{3}$ is the $o$-Ps lifetime expressed in nanoseconds, $R$ is the mean radius of the spherical well expressed in $\mathrm{nm}$ and $0.1656 \mathrm{~nm}$ is an empirical constant.

The results of our measurements suggest that there is no correlation between the temperature and $\tau_{1}$, and $I_{1}$ values. On the contrary, it seems that $\tau_{3}$ and $I_{3}$ values show some tendencies with temperature. The value of the longest lived component, $\tau_{3}$, vs. temperature is shown in Fig. 1 . The $\tau_{3}$ value decreases gradually with decreasing temperature, in the cooling cycle and increases in the heating cycle. It seems that there is no thermal hysteresis in the presented results. In spite of this, it seems that there is no simple correlation between the relative intensity of the longest lived component of the positron lifetimes, $I_{3}$, and temperature. The $I_{3}$ value as a function of temperature is presented in Fig. 2. It is absolutely clear that experimental points in the heating cycle do not follow the results observed in the cooling cycle. Going down from room to lower temperatures $I_{3}$ decreases first (to approximately $240 \mathrm{~K}$ ) and then it starts to gradually increase (in both the cooling and the heating cycles). Finally, the $I_{3}$ value, starts to decrease (in the heating cycle) for temperatures higher than about $140 \mathrm{~K}$.

Many authors observed a similar $\tau_{3}$ behaviour vs. temperature as we did for different polymers: [10-13] (their data in dark, as we performed our experiments in dark as well). 


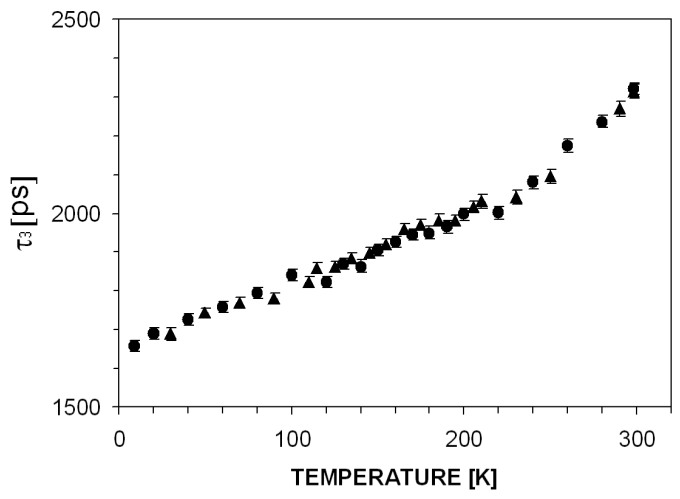

Fig. 1. $\tau_{3}$ values vs. temperature: $\bullet$ - cooling cycle, $\boldsymbol{\Delta}$ - heating cycle.

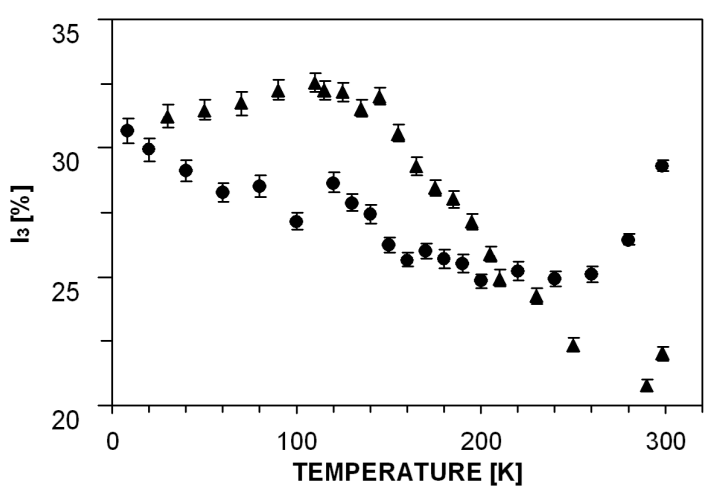

Fig. 2. $I_{3}$ values vs. temperature: $\bullet-$ cooling cycle, $\boldsymbol{\Delta}$ - heating cycle.

The relative intensity of the longest lived component, $I_{3}$, vs. temperature is more complex. It is well known and generally accepted that in a framework of the spur model of positron annihilation a large number of free electrons is produced in the sample irradiated by positrons. When a positron with a kinetic energy of several keV enters an absorber it quickly reaches thermal energies. It looses its energy mainly on exciting and ionisation of atoms and molecules of the absorber. The electrons released in this process might be trapped in shallow traps [14]. Free volume cavities may play a role of such traps in the polymer sample. During positron lifetime measurements in polymers, the number of electrons trapped in these traps increase, especially in low temperatures. In higher temperatures these electrons are detrapped. A similar explanation of the observed phenomena proposed Hirade et al. [15]. If it is so, the increasing number of the trapped electrons will cause the increase in the positronium formation probability. It might results in a larger value of $I_{3}$, during the heating cycle, from $8.3 \mathrm{~K}$ to about $140 \mathrm{~K}$. For the higher temperatures $I_{3}$ starts to drop as the result of detrapping of the electrons. Figure 2 shows these changes. 


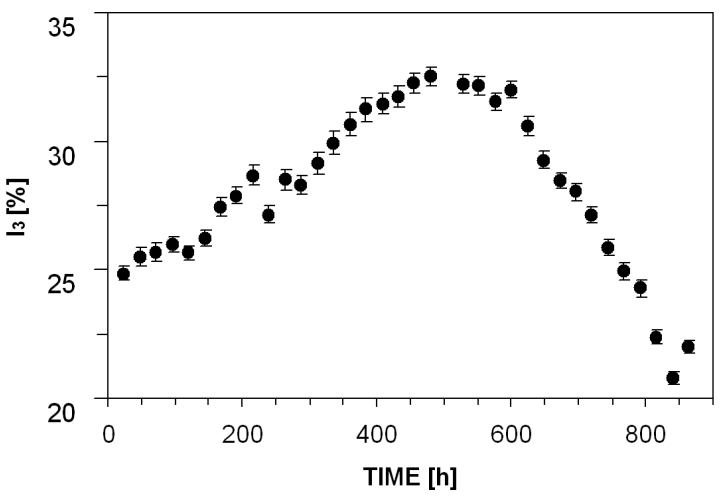

Fig. 3. $I_{3}$ values vs. elapsed time of the measurements.

From this figure one can find out that $I_{3}$ rises gradually with temperature from about $200 \mathrm{~K}$ to $8.3 \mathrm{~K}$ (in the cooling cycle) and it sill rises in the heating cycle from $8.3 \mathrm{~K}$ to about $150 \mathrm{~K}$. Though the temperature of these measurements was not constant, it was obviously low. We tried to look for the tendency of the $I_{3}$ values changes with time. Our attempt is presented in Fig. 3. The time values, as an independent variable, was estimated taking into account the elapsed time of the measurements. The time started to run, in Fig. 3, when the temperature of the investigated sample was $200 \mathrm{~K}$ (in the cooling cycle) and it ran till the end of the measurements. It seems that $I_{3}$ rises with the measurements time (or with keeping the sample in temperature below $200 \mathrm{~K}$ ). $I_{3}$ starts to decrease after about 600 hours of the measurements. After this time of the measurements the temperature of the sample reached about $150 \mathrm{~K}$ (in the heating cycle). The observed tendency of $I_{3}$ changes with time confirms the already mentioned mechanism of the formation of the electron traps in polymer in low temperature. The beginning of the decrease in the $I_{3}$ value at about $150 \mathrm{~K}$ might be explained as the result of detrapping of electrons from their shallow traps, due to molecular motion of the polymer molecules, which results in decreasing of the ortho-Ps formation probability. Quite similar behaviour of $I_{3}$ with time, in low but constant temperature, was observed by Chen et al. [11] and by He et al. [13] (data for the measurements in dark again).

\section{Conclusions}

In conclusion, we present the results of the positron lifetime measurements in polymethylpentene. At low temperature we observed the effect of trapping of the free electrons produced during positron irradiation. These electrons are trapped in free volume cavities resulting in the increase in the $o$-Ps formation probability. For the temperatures higher than about $150 \mathrm{~K}$, these electrons are detrapped and relative intensity of the longest lived positron lifetime component, $I_{3}$, decreases. 
This decrease might be explained by the motion of molecules of the polymer at the temperatures higher than about $150 \mathrm{~K}$.

\section{References}

[1] Y. Jean, Microchemical J. 42, 72 (1990).

[2] P.E. Mallon, in: Principles and Application of Positron and Positronium Chemistry, Eds. Y.C. Jean, P.E. Mallon, D.M. Schrader, World Scientific, Hong Kong 2003, p. 253.

[3] A. Danch, W. Osoba, Radiat. Phys. Chem. 63, 445 (2003).

[4] G. Charlet, G. Delmas, Polymer 25, 1619 (1984).

[5] A. Danch, J. Thermal Anal. 54, 151 (1998).

[6] A. Danch, J. Thermal Anal. 65, 525 (2001).

[7] P. Kirkegaard, N. Pedersen, M. Eldrup, PATFIT-88, Ris $\varnothing-M-2740$, Ris $\varnothing$ National Laboratory, Roskilde (Denmark) 1989.

[8] S. Tao, J. Chem. Phys. 56, 5499 (1972).

[9] M. Eldrup, D. Lightbody, J. Sherwood, Chem.Phys. 63, 51 (1981).

[10] J. Zrubcová, J. Krištiak, W. Pedersen, N. Pedersen, M. Eldrup, Mater. Sci. Forum 363-365, 359 (2001).

[11] Z. Chen, T. Suzuki, A. Uedono, S. Tanigawa, Y. Ito, Mater. Sci. Forum 363-365, 297 (2001)

[12] J. Krištiak, P. Bandžuch, O. Šauša, J. Zrubcowá, J. Bartoš, Mater. Sci. Forum 363-365, 269 (2001).

[13] C. He, T. Suzuki, L. Ma, M. Matsuo, V.P. Shantarovich, K. Kondo, Y. Ito, Phys. Lett. A 304, 49 (2002).

[14] R. Keyser, K. Tsuji, F. Williams, in: The Radiation Chemistry of Macromolecules, Ed. M. Mole, Academic Press, New York 1972, ch. 9.

[15] T. Hirade, F.H.J. Maurer, M. Eldrup, Radiat. Phys. Chem. 58, 465 (2000). 